\title{
Comparative study of bone mineral density, calcium, and vitamin D status in the Gujarati and white populations of Leicester
}

\author{
C Hamson, L Goh, P Sheldon, A Samanta
}

Postgrad Med J 2003;79:279-283

See end of article for authors' affiliations

Correspondence to: Dr A Samanta, Departmen of Rheumatology, Leicester Royal Infirmary, Leicester

LEI 5WW, UK ash.samanta@uhl-tr.nhs.uk

Submitted 12 July 2002 Accepted

25 November 2002

\begin{abstract}
Objectives: To evaluate differences in bone mineral density (BMD), calcium, and vitamin $D$ status between the Gujarati (South Asian) and white populations resident in Leicester and to determine whether this was linked to lifestyle factors.

Design: An observational cross sectional study of randomly selected Gujarati and white volunteers aged from $20-40$ years.

Setting: City of Leicester.

Participants: Subjects were randomly selected by age (20-40 years) and ethnicity. A total of 262 individuals volunteered to participate, of which 201 (51 white females, 71 Gujarati females, 37 white males, 42 Gujarati males) were eligible for the study.

Main outcome measures: Results of questionnaire, BMD at the hip and lumbar spine, and measurement of serum calcium, albumin, alkaline phosphatase, and 25 -hydroxyvitamin $\mathrm{D}$.

Results: Male and female white subjects were significantly taller and heavier than their Gujarati counterparts. There was a statistically significant difference in BMD both at the spine $(p<0.001)$ and hip $(p<0.001)$ between the white and Gujarati females with the Gujaratis having a lower BMD. There was a trend for Gujarati males to have a lower BMD at the hip and spine than their white counterparts but these figures did not reach statistical significance. The intensity of cigarette smoking and the amount of alcohol consumption were both higher in the white male and female subjects. Sunlight exposure (>4 hours per day) was significantly higher in white subjects compared with Gujaratis. There were no sig nificant differences in the mean level of serum calcium or alkaline phosphatase between the Gujaratis and whites. A significantly higher proportion $(p<0.001)$ of the Gujarati men and women had a vitamin $D$ level that was not measurable (that is, below the lower limit of the laboratory range of normal). Of those who had a measurable level (that is, in the normal range) mean levels of vitamin D were lower $(p<0.05)$ in the Gujarati men and women.

Conclusion: The present study is the first of its kind to note a low BMD in Gujarati subjects of South Asian origin compared with their white counterparts, living in Leicester. This study also confirms the presence of low serum vitamin D levels in Gujaratis. There is a need for more research in South Asians with regard to the collection of normal BMD values. This could provide a more meaningful reference range for identifying South Asians at risk of osteoporotic fractures and may have public health implications of relevance to this ethnic group.
\end{abstract}

ractures that might occur after minimal trauma in osteoporosis could have a considerable impact on health services. Early identification of subjects at risk followed by appropriate advice and intervention can help to minimise morbidity.

Identification of individuals at risk of osteoporosis according to the World Health Organisation (WHO) criteria requires accurate measurement of bone mineral density (BMD) and comparison to a young normal reference population. The WHO criteria for osteoporosis are based on a young adult normal white population as a reference point.

Over the last few years there has been a growing interest in the specific health care needs of ethnic minority groups. In Britain there is a diversity of ethnic origin in the overall population. There are approximately three million people of non-European origin, the majority being South Asians from the Indian subcontinent.

The population of Leicestershire comprises 24\% South Asians from the Indian subcontinent according to the 1991 census. The majority is from the Gujarat region in India (referred to as Gujaratis) who have migrated directly or indirectly to the UK.
There is a marked paucity of systematically collected data on BMD in South Asians. There is also a need for more ethnic group studies on BMD in specific ethnic groups, as this could subsequently have an impact on the delivery of health care in areas with high ethnic population. We have therefore performed an observational cross sectional study, measuring the BMD in randomly selected Gujaratis and white volunteers aged 20-40 years old. To our knowledge this study is the first of its kind.

The aim of this study was to compare BMD, calcium, and vitamin D status in the Gujarati and white populations resident within the city of Leicester and to assess a range of lifestyle variables in these two groups.

\section{SUBJECTS AND METHODS Background}

Ethical committee approval for this study was sought and obtained. The project was publicised by means of community

Abbreviations: $B M D$, bone mineral density; $\mathrm{WHO}$, World Health Organisation 
Table 1 Demographic features of subjects; values are mean (SE) unless stated otherwise

\begin{tabular}{|c|c|c|c|c|}
\hline & \multicolumn{2}{|l|}{ Males } & \multicolumn{2}{|l|}{ Females } \\
\hline & Whites $(n=37)$ & Gujaratis $(n=42)$ & Whites $(n=51)$ & Gujaratis $(n=71)$ \\
\hline Age (years) & $33(5)$ & $34(5)$ & $32(6)$ & $34(5)$ \\
\hline & & NS & & NS \\
\hline Weight (kg) & $86.3(19.2)$ & $\begin{array}{l}72.2(8.4) \\
(p<0.001)\end{array}$ & $68.2(12.7)$ & $\begin{array}{l}58.8(10.8) \\
(p<0.001)\end{array}$ \\
\hline Height (m) & $1.76(0.07)$ & $\begin{array}{l}1.69(0.06) \\
(p<0.01)\end{array}$ & $1.63(0.06)$ & $\begin{array}{l}1.55(0.05) \\
(p<0.001)\end{array}$ \\
\hline Proportion of subjects who were smokers (\%) & 35.1 & $\begin{array}{l}33.3 \\
\text { NS }\end{array}$ & 41.2 & $\begin{array}{l}2.8 \\
(p<0.001)\end{array}$ \\
\hline Amount smoked (number of pack years) & $16.3(11)$ & $\begin{array}{l}7.6(4.3) \\
(p<0.05)\end{array}$ & $13.0(10.2)$ & $\begin{array}{l}1.1(0.6) \\
(p<0.01)\end{array}$ \\
\hline Proportion of subjects who consumed alcohol (\%) & 97.3 & $\begin{array}{l}73.8 \\
(p<0.01)\end{array}$ & 88.2 & $\begin{array}{l}41.4 \\
(p<0.001)\end{array}$ \\
\hline Alcohol consumption (units per week) & $16.0(19.1)$ & $\begin{array}{l}10.1(12.8) \\
(p<0.01)\end{array}$ & $5.2(6.1)$ & $\begin{array}{l}1.8(1.5) \\
(p<0.001)\end{array}$ \\
\hline Use of oral contraceptive pills (\%) & - & - & 25.5 & $\begin{array}{l}15 \\
\text { NS }\end{array}$ \\
\hline Sunlight exposure (>4 hours per day) (\%) & 27 & $\begin{array}{l}11.9 \\
(p<0.001)\end{array}$ & 13.7 & $\begin{array}{l}7 \\
(p<0.01)\end{array}$ \\
\hline Non-vegetarian diet (\%) & 93.2 & $\begin{array}{l}70.2 \\
(p<0.05)\end{array}$ & 91.2 & $\begin{array}{l}50.7 \\
(p<0.001)\end{array}$ \\
\hline
\end{tabular}

centre presentations, an article in a local Gujarati newspaper, and by posters displayed in general practitioners' surgeries. Subjects who did not wish to be included in the study could ask to have their names deselected from recruitment.

\section{Subjects}

After permission from the Leicestershire Health Authority consenting practices were approached and every third name for both Asians and whites was targeted. A cross section of postcodes were covered in order to ensure a wide representation of socioeconomic groups. Potential candidates were initially selected by age (20-40 years) and presumed origin from Gujarat (the presence of a Gujarati surname). A total of 1358 letters were sent out over a period of 20 weeks. Invitation letters in both English and Gujarati were sent to each individual. Potential participants were asked to return a pre-printed reply slip and were provided with a stamped addressed envelope for this purpose. Positive replies were followed up to arrange a mutually convenient appointment time for measurement of BMD. A questionnaire that examined ethnic background, religion, smoking, alcohol intake, diet, and the use of oral contraceptives was sent to all volunteers before their appointments. The height and weight of subjects was recorded on the day of appointment.

\section{Measurement of BMD}

BMD was measured at both hip and lumbar region using a Lunar Expert-XL imaging densitometer. The Lunar Expert-XL has an integral hydroxyapatite standard that ensures that the systems remain calibrated over time. Deviations of more than $5 \%$ from expected values are highlighted in a daily quality assurance report which is performed to verify calibration. No deviation outside the normal range occurred during the course of this study. The expected precision error for the scanner is $0.01-0.03 \mathrm{~g} / \mathrm{cm}^{2}$. The effect of precision error was minimised by proper positioning of patients according to the manufacturer's guidelines, and by the use of scan mode.

\section{Biochemical analysis}

A sample of venous blood was collected from each volunteer for analysis of adjusted serum calcium, alkaline phosphatase, and serum 25-hydroxyvitamin D. The 25-hydroxyvitamin D levels for the two ethnic groups were collected in parallel over the same study period thus allowing for the seasonal variation in vitamin D levels. Vitamin D was analysed using DiaSorin 25-hydroxyvitamin D, 125-I radioimmunoassay kits. The DiaSorin 25-hydroxyvitamin D test procedure consists of two steps. The first involves extraction of 25-hydroxyvitamin D using acetonitrile. This is then assayed using a radioactive iodine procedure, which is based on a specific antibody to 25 -hydroxyvitamin D. The sample, antibody, and tracer are incubated for 90 minutes at $20-25^{\circ} \mathrm{C}$ with a second antibodyprecipitating complex. An additional buffer is also added after incubation to reduce non-specific binding. All subject samples together with five standard samples and a control sample were counted for one minute in a gamma scintillation counter. All samples were analysed in duplicate to ensure validity of the results.

Table 2 Biochemical features of subjects; values are mean (SE) unless stated otherwise

\begin{tabular}{|c|c|c|c|c|}
\hline & \multicolumn{2}{|l|}{ Males } & \multicolumn{2}{|l|}{ Females } \\
\hline & Whites & Gujaratis & Whites & Gujaratis \\
\hline Adjusted serum calcium $(2.12-2.65 \mathrm{mmol} / \mathrm{l})$ & $2.2(0.1)$ & $\begin{array}{l}2.2(0.1) \\
\text { NS }\end{array}$ & $2.2(0.088)$ & $\begin{array}{l}2.2(0.108) \\
\text { NS }\end{array}$ \\
\hline Serum alkaline phosphatase (25-110 IU/I) & $68.7(21)$ & $\begin{array}{l}75.0(19) \\
\text { NS }\end{array}$ & $57.4(13.4)$ & $\begin{array}{l}62.9(19.3) \\
\text { NS }\end{array}$ \\
\hline Mean vitamin D level for subjects who were within the normal range $(5-40 \mathrm{ng} / \mathrm{ml})$ & 14.8 & $\begin{array}{l}10.3 \\
(p<0.05)\end{array}$ & 13.0 & $\begin{array}{l}9.4 \\
(p<0.05)\end{array}$ \\
\hline $\begin{array}{l}\text { Proportion of subjects whose vitamin D level were below the lower limit of normal } \\
\text { (that is, } 5 \mathrm{ng} / \mathrm{ml})(\%)\end{array}$ & 3 & $\begin{array}{l}60 \\
(p<0.001)\end{array}$ & 0 & $\begin{array}{l}50.7 \\
(p<0.001)\end{array}$ \\
\hline
\end{tabular}




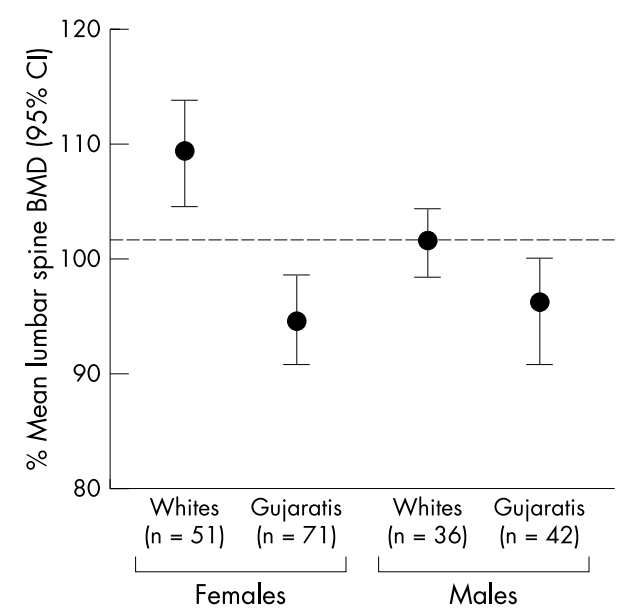

Figure 1 Mean lumbar spine BMD (95\% confidence interval).

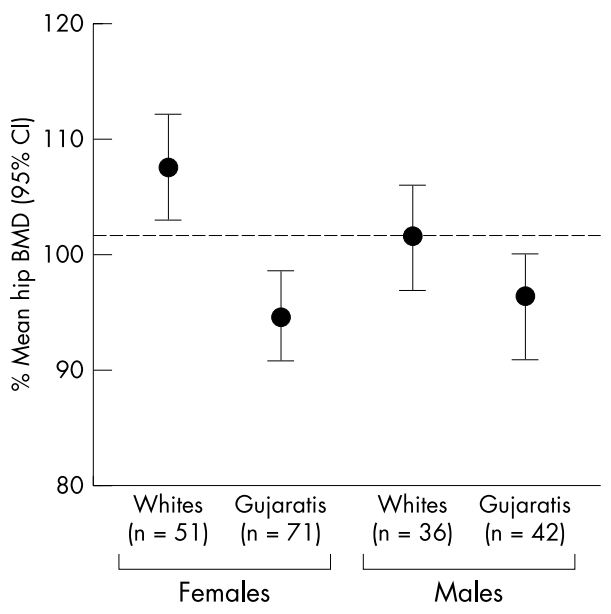

Figure 2 Mean hip BMD (95\% confidence interval).

\section{Statistical analysis}

All data collected (questionnaire responses, biochemical results, and bone densitometry measurements) was entered into Microsoft Excel file. These data were then numerically coded and transferred to SPSS (version 6, 1989-1993).

Mean and SD were calculated for all sets of data; $\chi^{2}$ test and independent sample $t$ test were applied to compare means between each ethnic group. The Mann-Whitney $U$ test was used to determine differences between data of mean ranked scores. Stepwise multiple linear regression was used to identify independent predictors of BMD at the hip and the spine for both genders in the two groups.

\section{RESULTS}

A total of 262 (19\% of total letters sent) individuals volunteered to participate in the study. Altogether 210 consenting adults eventually arrived for appointments; of these adults 201 (51 white female, 71 Gujaratis females, 37 white males, 42 Gujaratis males) were eligible for the study. Nine persons not of Gujarati origin or of mixed origin were excluded.

Table 1 shows the demographic features of subjects.

Both male and female white subjects had a significantly higher weight $(\mathrm{p}<0.001)$ and height $(\mathrm{p}<0.01)$ compared with their Gujarati counterparts. In addition the intensity of cigarette smoking and the amount of alcohol consumption was higher in the white group of both sexes. No significant differences were found in terms of the physical activity or socioeconomic classes, although a higher proportion of Gujarati males were professionals (35.7\% v 21.6\%). Sunlight exposure (in terms of the number of daylight hours spent outdoors) was significantly higher in white males and females compared with Gujaratis $(\mathrm{p}<0.01)$. A significantly higher proportion of white subjects included meat and fish in their diet compared with the Gujaratis (males $\mathrm{p}<0.05$; females $\mathrm{p}<0.001)$.

Table 2 shows the biochemical features of subjects. There were no significant differences in the mean level of serum calcium or alkaline phosphatase between the Gujaratis and whites. A significantly higher proportion $(p<0.001)$ of Gujarati men and women had a vitamin D level that was not measurable (that is, below the lower limit of the normal range). Of those who had a measurable level (that is, in the normal range), the mean levels of vitamin $\mathrm{D}$ were lower $(\mathrm{p}<0.05)$ in both Gujarati men and women.

Figures 1 and 2 show BMD at the lumbar spine and hip, respectively, as a percentage compared with the young adult reference range. The mean BMD at the spine in the Gujarati females was $1.11 \mathrm{~g} / \mathrm{cm}^{2}$ compared with $1.20 \mathrm{~g} / \mathrm{cm}^{2}$ in the white females $(\mathrm{p}<0.001)$. There were no statistically significant difference between males in the two groups (Gujaratis 1.17 $\mathrm{g} / \mathrm{cm}^{2}$; whites $1.23 \mathrm{~g} / \mathrm{cm}^{2}$ ).

The mean BMD measured at the hip in the Gujarati females was $0.94 \mathrm{~g} / \mathrm{cm}^{2}$ compared with $1.07 \mathrm{~g} / \mathrm{cm}^{2}$ in the white females $(\mathrm{p}<0.001)$. There were no statistical differences between the males in the two groups (Gujaratis $1.03 \mathrm{~g} / \mathrm{cm}^{2}$; whites 1.09 $\left.\mathrm{g} / \mathrm{cm}^{2}\right)$.

In summary, there was a statistically significant difference in BMD both at the spine and hip between the white and Gujarati females. There was no statistically significant difference in BMD between white and Gujarati males at the spine nor at the hip.

\begin{tabular}{|c|c|c|c|c|}
\hline \multirow[b]{2}{*}{ Variables } & \multicolumn{2}{|l|}{ Lumbar spine } & \multicolumn{2}{|l|}{ Hip } \\
\hline & $\begin{array}{l}\text { Coefficient of } \\
\text { variation (SE) }\end{array}$ & $\begin{array}{l}\text { Statistical } \\
\text { significance }\end{array}$ & $\begin{array}{l}\text { Coefficient of } \\
\text { variation (SE) }\end{array}$ & $\begin{array}{l}\text { Statistical } \\
\text { significance }\end{array}$ \\
\hline Ethnic origin & $-1.280(0.028)$ & $p<0.0001$ & $-0.091(0.023)$ & $p<0.0001$ \\
\hline Weight & $0.003(0.001)$ & $p<0.020$ & $0.004(0.001)$ & $p<0.0001$ \\
\hline Height & $0.113(0.103)$ & NS & $0.049(0.046)$ & NS \\
\hline Smoker & $-0.003(-0.163)$ & NS & $0.022(0.023)$ & NS \\
\hline Pack years & $0.159(0.162)$ & NS & $0.167(0.176)$ & NS \\
\hline Alcohol & $-0.158(-0.159)$ & NS & $-0.057(-0.060)$ & NS \\
\hline Units/week & $0.143(0.148)$ & NS & $0.095(0.102)$ & NS \\
\hline Sunlight exposure & $-0.086(-0.093)$ & NS & $0.037(0.041)$ & NS \\
\hline Meat in diet & $0.005(0.006)$ & NS & $-0.110(-0.118)$ & NS \\
\hline Fish in diet & $0.117(0.120)$ & NS & $-0.043(-0.045)$ & NS \\
\hline
\end{tabular}




\begin{tabular}{|c|c|c|c|c|}
\hline \multirow[b]{2}{*}{ Independent variables } & \multicolumn{2}{|l|}{ Lumbar spine } & \multicolumn{2}{|l|}{ Hip } \\
\hline & $\begin{array}{l}\text { Coefficient of } \\
\text { variation (SE) }\end{array}$ & $\begin{array}{l}\text { Statistical } \\
\text { significance }\end{array}$ & $\begin{array}{l}\text { Coefficient of } \\
\text { variation (SE) }\end{array}$ & $\begin{array}{l}\text { Statistical } \\
\text { significance }\end{array}$ \\
\hline Weight & $0.003(0.001)$ & $p<0.003$ & $0.005(0.001)$ & $p<0.0001$ \\
\hline Alcohol & $-0.101(0.046)$ & $p<0.032$ & $-0.196(-0.217)$ & NS \\
\hline Ethnic origin & $0.023(0.022)$ & NS & $0.026(0.026)$ & NS \\
\hline Height & $0.039(0.038)$ & NS & $0.022(0.022)$ & NS \\
\hline Pack years & $0.159(-0.003)$ & NS & $-0.133(-0.150)$ & NS \\
\hline Sunlight exposure & $0.073(0.079)$ & NS & $0.015(0.017)$ & NS \\
\hline Meat in diet & $-0.068(-0.070)$ & NS & $-0.060(-0.070)$ & NS \\
\hline Fish in diet & $0.015(0.015)$ & NS & $-0.054(0.060)$ & NS \\
\hline
\end{tabular}

Tables 3 and 4 show determinants of BMD in females and males respectively, analysed by stepwise multiple linear regression. For females, there was a significant correlation with ethnicity and weight for BMD at the lumbar spine and hip. For males, there was a significant correlation with weight alone for BMD at the hip, and with weight and alcohol consumption for BMD at the lumbar spine.

\section{DISCUSSION}

Ethnic variations in BMD are well documented with respect to black African, Hispanic, and white populations, but there are few data on South Asian populations. A study from the California, based on comparisons between Indian Asian and white women, found a lower BMD among Asians. ${ }^{1}$ However inferences drawn from these results could be limited by the fact that all the subjects had been referred for bone densitometry and could therefore have been selected on the basis of clinically suspicious low BMD.

There is a marked paucity of systematically collected data on BMD in South Asians in the UK. A study from Bradford measured BMD in a sample of 150 Indian women and compared this to age matched white controls. ${ }^{2}$ Preliminary results from this study indicated a lower BMD in Asians, which could have been due to small skeletal structure. ${ }^{3}$

In the present study we report significantly lower BMD at the hip and lumbar spine in Gujarati females compared with their white counterparts. There was a similar trend, although not statistically significant, for Gujarati males.

Although white females in this study appeared to have a high BMD this was only in relation to the young female reference population. There was no significant difference in the absolute values of BMD between white females and males.

By multiple regression analysis, body weight appeared to be the most important predictor of BMD for the current sample. For both genders a positive correlation was found between BMD and weight. The relationship between BMD and weight is not necessary causal as a high BMD will itself give rise to an increased weight. On the other hand, increased weight leads to increase mechanical stresses on the bones, which in turn affect the remodelling process. Lean body mass and degree of adiposity are components of body weight, both of which may confer protection from fracture.

The lower BMD in the Gujarati population could be due to arthropometric differences such as bone size. However height was not significantly associated with low BMD in Gujaratis on multiple regression analysis.

For males, alcohol consumption was positively associated with high BMD. This is contrary to evidence that high alcohol intake is associated with low BMD. Some authors, however, suggested that a moderate alcohol intake has little effect on bones. It is possible that the relationship between BMD and alcohol intake follows a J-shaped curve, with moderate alcohol intake actually improving BMD. ${ }^{45}$ Increased body weight directly due to alcohol ingestion was included in the model for regression analysis.

The cause of the observed ethnic difference in BMD may be genetic or environmental. Seventy five percent of peak bone mass is thought to be genetically determined. ${ }^{6}$ Evidence from a review of hereditary versus environmental influences on BMD provides support for a hereditary component in determining peak BMD in young adult women. ${ }^{7}$ However, if this is true, it would be difficult to understand why this is expressed more prominently in females compared with males. An evaluation of genetic factors influencing ethnic variations in BMD was outside the scope of this study. Environmental factors such as dietary intake, physical activity, and hormonal factors have also been documented to exert an effect on BMD, although the effect is much less. Environmental influences could be important in the present sample and could be due to culturally determined differences between the groups such as the amount of sunlight exposure. It is not possible to comment on the effect of dress preference (the use of traditional "burkhas" in Gujarati women) as a contributory factor on BMD as these data were not collected.

Smoking and alcohol consumption appear to indicate greater westernisation in Gujarati males. Acculturation in Gujarati males could possibly be an environmental influence that could account for the lack of any significant difference in BMD when compared with white males.

Nutrition is influential to the acquisition of bone mass. ${ }^{8}$ Dietary anomalies, particularly in calcium intake, have been implicated in the geographical variations of adult BMD. ${ }^{9}$ Low serum 25-hydroxyvitamin D has also been previously seen to contribute to low BMD at the lumbar spine and neck of femur for post-menopausal women, ${ }^{10}$ although it is less clear whether 25-hydroxyvitamin D regulates peak bone mass as well as bone loss. Our study sample revealed a low concentration of serum vitamin D in $50 \%-60 \%$ of the Gujarati subjects. It is possible that the normal range for vitamin $\mathrm{D}$ is lower in Gujaratis because the Gujarati subjects in our group did not have any clinical or biochemical features of osteomalacia. There were no abnormalities in bone biochemistry in the subgroup of patients with a low vitamin D in comparison with those subjects with normal vitamin $\mathrm{D}$, although data on parathyroid hormone were not available in the study subjects. In multiple linear regression analysis on BMD as the dependent variable, when ethnicity, weight, and alcohol consumption were taken into account, vitamin D levels categorised as normal or low were not found to be independent predictors of BMD. This would rebut underlying osteomalacia as an explanation for the lower BMD in Gujaratis.

The present study, which is the first of its kind, has noted low BMD in Gujaratis in Leicester. This paper also shows an increased prevalence of low serum vitamin D in this group of South Asians and adds to the sparse data on BMD in this 
population in the UK. There is currently little evidence based on research to give any real understanding of the problem of osteoporosis among South Asians living in Britain. The current WHO criteria for the diagnosis of osteoporosis is based on a normal adult white population. These criteria cannot necessarily be extrapolated to ethnic populations. There is a need for more research to establish bone density norms for males and females of different age groups within the South Asian population and to reference this against T-scores. This is important in arriving at a clinician-orientated decision as to whether or not to treat subjects at risk of developing fractures, ${ }^{11}$ thus implementing useful preventive measures before fractures occur. The present study may also have implications for public health. As the Gujarati population of Leicester ages, low BMD in the presence of a small skeletal stature might be associated with increased risk of fractures. If this should be the case, then appropriate interventional measures will be required to address this particular health issue in South Asians.

\section{Authors' affiliations}

C Hamson, L Goh, P Sheldon, A Samanta, Department of Rheumatology, University Hospitals of Leicester NHS Trust, Leicester Royal Infirmary

\section{REFERENCES}

1 Bhudhikanok GS, Wang MC, Eckert K, et al. Differences in bone mineral in young Asians and Caucasian Americans may reflect differences in bone size. J Bone Miner Res 1996;11:1545-56.

2 Delvin J, Armes FM, Rajaratnam G, et al. Bone mineral density amongst Asian women in Bradford. In: Ring EFJ, Elvins DM, Bhalla AK, eds. Current research in osteoporosis and bone mineral measurement 5. London: British Institute of Radiology, 1998: 37-8.

3 Russel-Aulet $M$, Wang J, Thornton JC, et al. Bone mineral density and mass in a cross-sectional study of white and Asian women. J Bone Miner Res 1993:8:575-82.

4 TNO Nutrition and Food Research Institute. The western diet with a special focus on dairy products. Ghent: TNO Nutrition and Food Research Institute, 1995.

5 Heaney RP. Bone mass, nutrition, and other lifestyle factors. Am J Med 1993;95:29S-33.

6 Cooper C. Epidemiology and definition of osteoporosis. In: Compston $\mathrm{JE}$, ed. Osteoporosis, new perspectives on causes, prevention and treatment. London: RCP, 1996: 1-10.

7 Pollitzer WS, Anderson JJB. Ethnic and genetic differences in bone mass. A review with a hereditary versus environmental perspective. Am J Clin Nutr 1989:50:1244-59.

8 Vaishnava H, Rizvi S. Frequency of osteomalacia and osteoporosis in fractures of the proximal femur. Lancet 1974;i:676-7.

9 Matkovic V, Kositial K, Simonovic l, et al. Bone status and fracture rates in two regions of Yugoslavia. Am J Clin Nutr 1979;32:540-9.

10 Collins D, Jasani C, Fogelman I. Vitamin D and bone mineral density. Osteoporos Int 1998;8:110-4

11 Dequeker J. Osteoporotic fractures, aging and bone density T-score. Clin Rheumatol 2000;19:171-3. 